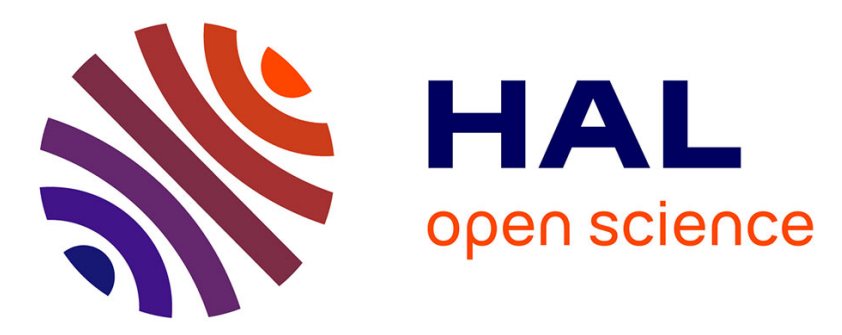

\title{
Stability of a Kirchhoff-Roe scheme for two-dimensional linearized Euler systems
}

Emmanuel Franck, Laurent Gosse

\section{To cite this version:}

Emmanuel Franck, Laurent Gosse. Stability of a Kirchhoff-Roe scheme for two-dimensional linearized Euler systems. Annali dell'Universita di Ferrara, 2017, pp.1-26. 10.1007/s11565-017-0296-9 . hal01666060

\section{HAL Id: hal-01666060 https://hal.science/hal-01666060}

Submitted on 26 Jan 2018

HAL is a multi-disciplinary open access archive for the deposit and dissemination of scientific research documents, whether they are published or not. The documents may come from teaching and research institutions in France or abroad, or from public or private research centers.
L'archive ouverte pluridisciplinaire HAL, est destinée au dépôt et à la diffusion de documents scientifiques de niveau recherche, publiés ou non, émanant des établissements d'enseignement et de recherche français ou étrangers, des laboratoires publics ou privés. 


\title{
Stability of a Kirchhoff-Roe scheme for two-dimensional linearized Euler systems
}

\author{
Emmanuel Franck • Laurent Gosse
}

the date of receipt and acceptance should be inserted later

\begin{abstract}
By applying Helmholtz decomposition, the unknowns of a linearized Euler system can be recast as solutions of uncoupled linear wave equations. Accordingly, the Kirchhoff expression of the exact solutions is recast as a timemarching, Lax-Wendroff type, numerical scheme for which consistency with one-dimensional upwinding is checked. This discretization, involving spherical means, is set up on a 2D uniform Cartesian grid, so that the resulting numerical fluxes can be shown to be conservative. Moreover, semi-discrete stability in the $H^{s}$ norms and vorticity dissipation are established, along with practical second-order accuracy. Finally, some relations with former "shape functions" and "symmetric potential schemes" are highlighted.
\end{abstract}

Keywords Linearized Euler system · Kirchhoff exact solution · 2D LaxWendroff scheme $\cdot$ Numerical vorticity dissipation

Mathematics Subject Classification (2000) 65M06 - 65M12 - 35L45 · 35L05 - 35Q05

\section{Introduction and modeling}

In order to solve a multi-dimensional system of inviscid compressible Euler equations, splitting between acoustic and transport processes can be viewed as an appealing strategy, see e.g. [29, Part II] and $[17,30]$.

1.1 Reduction of a linearized inviscid Euler system to wave equations

The inviscid Euler system, linearized around null velocity, reads, for $\mathbf{x} \in \mathbb{R}^{3}$,

$$
\forall t>0, \quad \partial_{t} p(t, \mathbf{x})+\operatorname{div}(\mathbf{U})=0, \quad \partial_{t} \mathbf{U}(t, \mathbf{x})+\nabla(p)=\mathbf{F}(\mathbf{x}) .
$$

INRIA, 7 Rue René Descartes, 67000 Strasbourg, France E-mail: emmanuel.franck@inria.fr . Istituto per le Applicazioni del Calcolo, via dei Taurini, 19, 00185 Rome, Italy E-mail: l.gosse@ba.iac.cnr.it 
By applying Helmholtz decomposition to both $\mathbf{F}$ and $\mathbf{U}$, yields,

$$
\begin{aligned}
\forall \mathbf{x} \in \mathbb{R}^{3}, \quad \mathbf{F}(\mathbf{x})=\nabla \Phi(\mathbf{x})+\operatorname{rot} \mathbf{G}(\mathbf{x}), \quad \Delta \Phi=\operatorname{div}(\mathbf{F}), \\
\forall t>0, \mathbf{x} \in \mathbb{R}^{3}, \quad \mathbf{U}(t, \mathbf{x})=\mathbf{u}(t, \mathbf{x})+\mathbf{v}(t, \mathbf{x}), \quad \operatorname{div}(\mathbf{v}) \equiv 0 .
\end{aligned}
$$

- The fluctuations of $p(t, \mathbf{x})$ clearly satisfy a wave equation,

$$
\partial_{t t} p-\Delta p=\Delta \Phi, \quad \partial_{t t} \tilde{p}-\Delta \tilde{p}=0, \text { for } \tilde{p}=p-\Phi .
$$

- Initial vorticity evolves only through $\mathbf{G}(\mathbf{x})$ :

$$
\partial_{t}(\operatorname{rot} \mathbf{U})+\operatorname{rot}(\nabla p)=\partial_{t}(\operatorname{rot} \mathbf{v})=\operatorname{rot}(\operatorname{rot} \mathbf{G})
$$

so that,

$$
\operatorname{rot}\left(\partial_{t} \mathbf{v}-\operatorname{rot} \mathbf{G}(\mathbf{x})\right)=0, \quad \text { in particular, } \operatorname{rot}\left(\partial_{t t} \mathbf{v}\right) \equiv 0
$$

But since $\operatorname{div}\left(\partial_{t t} \mathbf{v}\right)=\partial_{t t}(\operatorname{div} \mathbf{v}) \equiv 0$ by definition of $\mathbf{v}$, it follows that

$$
\forall t>0, \mathbf{x} \in \mathbb{R}^{3}, \quad \partial_{t t} \mathbf{v}(t, \mathbf{x})=0 .
$$

- Yet differentiate in time the impulsion equation,

$$
\partial_{t t} \mathbf{U}+\nabla\left(\partial_{t} p\right)=0, \quad \frac{\partial \mathbf{F}}{\partial t} \equiv 0,
$$

but since $\operatorname{div}(\mathbf{U})=\operatorname{div}(\mathbf{u})$ by Helmholtz decomposition, it follows that

$$
\partial_{t} p+\nabla(\operatorname{div}(\mathbf{u}))=0, \quad \text { so that } \partial_{t t}(\mathbf{u}+\mathbf{v})-\boldsymbol{\Delta} \mathbf{u}=0
$$

with $\boldsymbol{\Delta}(\cdot)$ the usual vector-Laplacian of a curl-free vector field.

Finally we reach a decoupled system of inhomogeneous 3D wave equations,

$$
\begin{aligned}
\partial_{t t} p-\Delta p & =\Delta \Phi(\mathbf{x}), \\
\partial_{t t} \mathbf{u}-\Delta \mathbf{u} & =0 \\
\partial_{t} \mathbf{v}=(\mathbf{F}-\nabla \Phi), \quad \partial_{t t} \mathbf{v} & =0
\end{aligned}
$$

Even if $\mathbf{F} \equiv 0$, the derivation of a reliable, genuinely multi-dimensional numerical scheme for a 3D wave equation is important for solving the Cauchy problem for (1.1) in a context of smooth solutions, written in the form (1.2). 
1.2 Elementary a-priori $H^{s}$ estimates in $2 \mathrm{D}$

System (1.1) rewrites, for $\mathbf{x}=(x, y) \in \mathbb{R}^{2}$ and $\mathbf{V}=(p, \mathbf{U}) \in \mathbb{R}^{3}$, as

$$
\partial_{t} \mathbf{V}+A \partial_{x} \mathbf{V}+B \partial_{x} \mathbf{V}=0, \quad A=\left(\begin{array}{lll}
0 & 1 & 0 \\
1 & 0 & 0 \\
0 & 0 & 0
\end{array}\right), B=\left(\begin{array}{lll}
0 & 0 & 1 \\
0 & 0 & 0 \\
1 & 0 & 0
\end{array}\right)
$$

As both $A, B$ are symmetric matrices, results in that,

$$
A B=A^{T} B^{T}=(B A)^{T} \neq B A \text {, because } B A \text { is not diagonal, }
$$

so that $L^{1}$ and $B V$ estimates shouldn't be expected to hold for (1.1). Indeed,

Theorem 1.1 Assume initial data $\mathbf{V}_{0}(\mathbf{x})=\left(p_{0}(\mathbf{x}), u(\mathbf{x}), v(\mathbf{x})\right) \in H^{s}\left(\mathbb{R}^{2}\right)^{3}$ for $s \in \mathbb{N}$, where $H^{0}=L^{2}$, then, for any $t>0, \mathbf{V}(t, x, y) \in H^{s}\left(\mathbb{R}^{2}\right)^{3}$.

Proof Being (1.1) linear and translation-invariant, it suffices to prove the statement for $s=0$, a $L^{2}$ uniform bound. Multiplying (1.1) by $(p, u, v)$ yields

$$
\frac{1}{2} \partial_{t}\left(p^{2}+u^{2}+v^{2}\right)+\partial_{x}(p u)+\partial_{y}(p v)=0,
$$

and by integration in $\mathbb{R}^{2}$,

$$
E^{\prime}(t):=\frac{d}{d t} \int_{\mathbb{R}^{2}}|p|^{2}(t, \mathbf{x})+|\mathbf{U}|^{2}(t, \mathbf{x}) d \mathbf{x}=0
$$

Following Rauch [24], BV-estimates don't hold for (1.1). However, dimensionalsplitting algorithms, alternatively solving $1 \mathrm{D}$ systems [25], preserve (or numerically dissipate, because of artificial viscosity) all the $L^{p}$ norms, $1 \leq p \leq+\infty$ :

$$
\left\{\begin{array}{rl}
\partial_{t} f^{ \pm} \pm \partial_{x} f^{ \pm} & =0, \\
\partial_{t} v & =0, \\
f^{ \pm} & =u \pm p,
\end{array}, \quad\left\{\begin{aligned}
\partial_{t} g^{ \pm} \pm \partial_{y} g^{ \pm} & =0 \\
\partial_{t} u & =0 \\
g^{ \pm} & =v \pm p
\end{aligned}\right.\right.
$$

so that, any one-directional step necessarily "undoes" part of what the previous step did before, simply because in the limit, only the $L^{2}$-energy dissipation (1.3) holds.

\subsection{Aims and plan of the paper}

Motivated by (1.1), we derive a multi-dimensional scheme, based on Kirchhoff's formula for the linear wave equation,

$$
\partial_{t t} \phi-\Delta \phi=0, \quad \phi(t=0, \mathbf{x})=\phi_{0}(\mathbf{x}), \quad \partial_{t} \phi(t=0, \mathbf{x})=\partial_{t} \phi_{0}(\mathbf{x}) .
$$

It's not the first time that Kirchhoff's explicit expression is used for improving multi-dimensional features of numerical schemes, see e.g. [2-4], in order to 
circumvent the complexity of 2D Riemann problems, [17]. For instance, the 2D Riemann problem for (1.1) was explicitly solved in [1, Chap. 6] for $\mathbf{F} \equiv 0$. Other attempts to go beyond usual dimensional-splitting strategies were reported in e.g. [ $6,10,16,23,25,28]$, along with "vorticity-preserving" algorithms, $[13,20,15,21]$. Here, our aim is to follow the insight of [8], that is, to reformulate Kirchhoff's expression into a semi-discrete (in space) Lax-Wendroff type time-marching scheme [14]. On a uniform 2D Cartesian grid, it turns out that the algorithm resulting from discretizing all the remaining terms matches the one written in [20, eqn. (3.12)] when $\Delta x=\Delta y$ (which is compatible with rotational invariance of the continuous equations). The idea of building numerical schemes, supposedly keeping multi-dimensional interactions, starting from the expression of (locally) exact solutions is reminiscent of e.g. [9,11].

In Section 3, the passage from Kirchhoff's formula to a time-marching scheme is detailed in Theorem 2.1 for which a one-dimensional reduction is studied. Discrete numerical fluxes are derived in Section 4, for which semidiscrete (in time) stability estimates are established in Section 5. Numerical assessments (especially, second-order accuracy on smooth solutions) are presented in Section 6. At last, some relations are drawn with previous constructions, especially the "shape functions" of [13], and [5].

\section{Derivation of Kirchhoff-based numerical scheme}

2.1 Kirchhoff's formula as a Lax-Wendroff scheme

By defining the so-called "spherical mean" (or average) of a function $f$ as,

$$
\forall r>0, \quad M_{r}\{f\}(\mathbf{x})=\frac{1}{4 \pi r^{2}} \int_{\partial B_{r}(\mathbf{x})} f(\sigma) d \sigma,
$$

and assuming $C^{2}$ smoothness on initial data to ensure that,

$$
\forall \mathbf{x} \in \mathbb{R}^{3}, \quad M_{r}\{\phi\}(t, \mathbf{x}) \rightarrow \phi(t, \mathbf{x}) \text { as } r \rightarrow 0,
$$

along with the same continuity property for both $\partial_{t} \phi$ and $\Delta \phi$, one proves Kirchhoff's representation formula, see [7, page 77], of $\phi(t, \mathbf{x})$ :

$$
\forall t>0, \quad \phi(t, \mathbf{x})=\partial_{t}\left(t \cdot M_{t}\left\{\phi_{0}\right\}(\mathbf{x})\right)+t \cdot M_{t}\left\{\partial_{t} \phi_{0}\right\}(\mathbf{x}) .
$$

An insightful observation [8] is that (2.2) recasts as a Lax-Wendroff scheme:

Theorem 2.1 The exact (smooth enough) solution of the Cauchy problem for the $3 D$ wave equation (1.4) with data $\phi_{0}$ and $\partial_{t} \phi_{0}$ reads: $\forall \mathbf{x} \in \mathbb{R}^{3}, t>0$,

$$
\phi(t, \mathbf{x})=\phi_{0}(\mathbf{x})+t \cdot M_{t}\left\{\partial_{t} \phi_{0}\right\}(\mathbf{x})+\int_{0}^{t} \tau M_{\tau}\left\{\Delta \phi_{0}\right\}(\mathbf{x}) d \tau .
$$


In order to derive a time-marching numerical scheme involving artificial viscosity, one needs the following lemma, which is a chain-rule type of result:

Lemma 2.1 (admitted, see e.g. Evans [7]) For any smooth function $f$,

$$
\frac{\partial}{\partial r} M_{r}\{f\}(\mathbf{x})=\frac{1}{4 \pi r^{2}} \int_{\partial B_{r}(\mathbf{x})} \frac{\partial f}{\partial \mathbf{n}}(\sigma) d \sigma=\frac{1}{4 \pi r^{2}} \int_{B_{r}(\mathbf{x})} \Delta f\left(\mathbf{x}^{\prime}\right) d \mathbf{x}^{\prime} .
$$

Moreover, there holds the so-called "Euler-Darboux identity",

$$
\forall r>0, \mathbf{x} \in \mathbb{R}^{3}, \quad \frac{\partial}{\partial r}\left(r^{2} \frac{\partial}{\partial r} M_{r}\{f\}(\mathbf{x})\right)=r^{2} M_{r}\{\Delta f\}(\mathbf{x}) .
$$

Proof By expanding the time-derivative in (2.2) and using the chain-rule,

$$
\frac{\partial}{\partial t} M_{t}\left\{\phi_{0}\right\}(\mathbf{x})=\frac{1}{4 \pi t^{2}} \int_{B_{t}(\mathbf{x})} \Delta \phi_{0}\left(\mathbf{x}^{\prime}\right) d \mathbf{x}^{\prime}=\frac{1}{4 \pi t^{2}} \int_{0}^{t}\left(\int_{\partial B_{\tau}(\mathbf{x})} \Delta \phi_{0}(\sigma) d \sigma\right) d \tau .
$$

So, the $4 \pi$ cancel each other by letting $M_{t}\left\{\Delta \phi_{0}\right\}$ appear,

$$
\phi(t, \mathbf{x})=M_{t}\left\{\phi_{0}\right\}(\mathbf{x})+t \cdot M_{t}\left\{\partial_{t} \phi_{0}\right\}(\mathbf{x})+\frac{1}{t} \int_{0}^{t} \tau^{2} M_{\tau}\left\{\Delta \phi_{0}\right\}(\mathbf{x}) d \tau .
$$

Yet, in order to reach (2.3), one must relate $M_{t}\left\{\phi_{0}\right\}(\mathbf{x})$ and $\phi_{0}(\mathbf{x})$. However,

$$
\forall t>0, \quad \phi_{0}(\mathbf{x})=M_{t}\left\{\phi_{0}\right\}(\mathbf{x}) \Rightarrow \phi_{0} \text { is harmonic. }
$$

By integrating once the Euler-Darboux identity,

$$
\forall t>0, \quad t \frac{\partial}{\partial t} M_{t}\left\{\phi_{0}\right\}(\mathbf{x})=\frac{1}{t} \int_{0}^{t} \tau^{2} M_{\tau}\left\{\Delta \phi_{0}\right\}(\mathbf{x}) d \tau .
$$

Moreover, by assuming enough smoothness,

$$
\begin{aligned}
t M_{t}\left\{\Delta \phi_{0}\right\}(\mathbf{x}) & =t \frac{\partial^{2}}{\partial t^{2}} M_{t}\left\{\phi_{0}\right\}(\mathbf{x})+2 \frac{\partial}{\partial t} M_{t}\left\{\phi_{0}\right\}(\mathbf{x}) \\
& =\frac{\partial}{\partial t}\left(t \frac{\partial}{\partial t} M_{t}\left\{\phi_{0}\right\}(\mathbf{x})\right)+\frac{\partial}{\partial t} M_{t}\left\{\phi_{0}\right\}(\mathbf{x})
\end{aligned}
$$

which can be integrated in $(0, t)$ in order to produce,

$$
t \frac{\partial}{\partial t} M_{t}\left\{\phi_{0}\right\}(\mathbf{x})+M_{t}\left\{\phi_{0}\right\}(\mathbf{x})-M_{0}\left\{\phi_{0}\right\}(\mathbf{x})=\int_{0}^{t} \tau M_{\tau}\left\{\Delta \phi_{0}\right\}(\mathbf{x}) d \tau .
$$

By regularity, $M_{0}\left\{\phi_{0}\right\}(\mathbf{x})=\phi_{0}(\mathbf{x})$ for any $\mathbf{x} \in \mathbb{R}^{3}$, so that,

$$
M_{t}\left\{\phi_{0}\right\}(\mathbf{x})=\phi_{0}(\mathbf{x})-\frac{1}{t} \int_{0}^{t} \tau^{2} M_{\tau}\left\{\Delta \phi_{0}\right\}(\mathbf{x}) d \tau+\int_{0}^{t} \tau M_{\tau}\left\{\Delta \phi_{0}\right\}(\mathbf{x}) d \tau
$$

which can be plugged into (2.4) in order to reach (2.3) by cancellation. 
2.2 Notion of consistency

Consistency of (2.3) with the wave equation (1.4) proceeds by rewriting the resulting Lax-Wendroff scheme in the following form,

$$
\frac{\phi(t, \mathbf{x})-\phi_{0}(\mathbf{x})}{t}-M_{t}\left\{\partial_{t} \phi_{0}\right\}(\mathbf{x})=\frac{1}{t} \int_{0}^{t} \tau M_{\tau}\left\{\Delta \phi_{0}\right\}(\mathbf{x}) d \tau,
$$

in order to see that, for smooth $\phi$ and any $\mathbf{x}$, when $t \rightarrow 0^{+}$,

$$
\begin{aligned}
\frac{\phi(t, \mathbf{x})-\phi_{0}(\mathbf{x})}{t} & \rightarrow \partial_{t} \phi(t, \mathbf{x}), \\
M_{t}\left\{\partial_{t} \phi_{0}\right\}(\mathbf{x})=\partial_{t} \phi_{0}(\mathbf{x})+t^{2} \Delta_{\mathbf{x}}\left(\partial_{t} \phi_{0}\right)(\mathbf{x}) / 6+O\left(t^{4}\right) & \rightarrow \partial_{t} \phi_{0}(\mathbf{x}), \\
\frac{1}{t} \int_{0}^{t} \tau M_{\tau}\left\{\Delta \phi_{0}\right\}(\mathbf{x}) d \tau & \rightarrow t \cdot \Delta \phi_{0}(\mathbf{x}),
\end{aligned}
$$

so that, by dividing again by $t$, the wave equation (1.4) emerges.

\section{Application of the Kirchhoff-based scheme to wave-systems}

3.1 Multi-dimensional features lie in spherical means

The scheme (2.3) applies in a straightforward manner to pressure fluctuations in $(1.2)$,

$$
p(\Delta t, \mathbf{x})=p_{0}(\mathbf{x})-\Delta t \cdot M_{\Delta t}\left\{\operatorname{div} \mathbf{u}_{0}\right\}(\mathbf{x})+\int_{0}^{\Delta t} \tau M_{\tau}\left\{\Delta p_{0}\right\}(\mathbf{x}) d \tau
$$

thanks to the continuity equation, $\partial_{t} p=-\operatorname{div}(\mathbf{u})$. The vector-Laplacian has uncoupled components, so that (2.3) applies to the curl-free vector $\mathbf{u}$, too,

$$
\mathbf{u}(\Delta t, \mathbf{x})=\mathbf{u}_{0}(\mathbf{x})-\Delta t \cdot M_{\Delta t}\left\{\nabla p_{0}\right\}(\mathbf{x})+\int_{0}^{\Delta t} \tau M_{\tau}\left\{\boldsymbol{\Delta} \mathbf{u}_{0}\right\}(\mathbf{x}) d \tau,
$$

after having inserted the impulsion equation $\partial_{t} \mathbf{u}=-\nabla p$. Now, it is interesting to consider (3.1)-(3.2) inside which one decides to impose,

$$
M_{\Delta t}\{\cdot\} \rightarrow M_{0}\{\cdot\}, \quad M_{\tau}\{\cdot\} \rightarrow M_{0}\{\cdot\},
$$

that is to say, one gets rid of all the spherical averaging process. Then,

$$
\begin{aligned}
& p(\Delta t, \mathbf{x})=p_{0}(\mathbf{x})-\Delta t \cdot \operatorname{div}\left(\mathbf{u}_{0}\right)(\mathbf{x})+\frac{\Delta t^{2}}{2} \cdot \Delta p_{0}(\mathbf{x}) \\
& \mathbf{u}(\Delta t, \mathbf{x})=\mathbf{u}_{0}(\mathbf{x})-\Delta t \cdot \nabla p_{0}(\mathbf{x})+\frac{\Delta t^{2}}{2} \cdot \Delta \mathbf{u}_{0}(\mathbf{x})
\end{aligned}
$$

which matches the usual dimensional-splitting scheme if, on a given uniform Cartesian grid, the operators $\nabla$, div, and $\Delta$ are discretized with centered finitedifferences. So we are led to finding reliable quadrature formulas for a practical computation of averages $M_{t}\{\cdot\}$ in order to take full advantage of (3.1)-(3.2) in a multi-dimensional context. 
3.2 One-dimensional reduction and upwinding

Suppose that $\mathbf{F} \equiv 0$ and given translation-invariant Cauchy-data like,

$$
p(t=0, \mathbf{x})=p_{0}(x), \quad \mathbf{u}(t=0, \mathbf{x})=\left(u_{0}(x), 0\right), \quad \mathbf{v} \equiv 0,
$$

then (1.2) propagate this invariance in time and (1.1) reduces to,

$$
\partial_{t} p+\partial_{x} u=0, \quad \partial_{t} u+\partial_{x} p=0 .
$$

In $1 \mathrm{D}$, the average (2.1) for any $r>0$ is just,

$$
\forall x \in \mathbb{R}, \quad M_{r} f(x)=\frac{1}{2 r} \int_{-r}^{r} f(x+s) d s,
$$

so that applying the scheme (2.3) to the resulting system yields,

$$
\begin{aligned}
\Delta t \cdot M_{\Delta t}\left\{\partial_{x} u\right\}(\mathbf{x}) & =\frac{\Delta t}{2 \Delta t} \int_{-\Delta t}^{\Delta t} \partial_{x} u(x+s) d s \\
& =\frac{\Delta t}{2 \Delta x}(u(x+\Delta x)-u(x-\Delta x)), \quad \text { for } \Delta t=\Delta x,
\end{aligned}
$$

which a centered difference, along with, (again for $\Delta t=\Delta x$ )

$$
\begin{aligned}
\int_{0}^{\Delta t} \tau M_{\tau}\left\{\Delta p_{0}\right\}(\mathbf{x}) d \tau & =\int_{0}^{\Delta t} \frac{\tau}{2 \tau} \int_{-\tau}^{\tau} \partial_{x x} p(x+s) d s d \tau \\
& =\frac{1}{2} \int_{0}^{\Delta t}\left(\partial_{x} p(x+\tau)-p(x-\tau)\right) d \tau \\
& =\frac{\Delta t}{2 \Delta x}(p(x+\Delta x)-2 p(x)+p(x-\Delta x))
\end{aligned}
$$

which is a standard numerical viscosity term. Since in $1 \mathrm{D}$, the velocity $u(x)$ is a scalar, too, the corresponding discretization is identical. By adding and subtracting $p$ and $u$ in order to form diagonal variables (Riemann invariants) $f^{ \pm}=u \pm p$, one recovers the standard upwind form,

$$
\begin{aligned}
& f^{+}(t, x)=f^{+}(0, x)-\frac{\Delta t}{\Delta x}\left(f^{+}(0, x)-f^{+}(0, x-\Delta x)\right), \\
& f^{-}(t, x)=f^{-}(0, x)-\frac{\Delta t}{\Delta x}\left(f^{-}(0, x+\Delta x)-f^{-}(0, x)\right) .
\end{aligned}
$$

In $\Delta t=\Delta x$, there is no numerical viscosity, so one recovers the exact solution,

$$
\forall t, x \in \mathbb{R}^{2}, \quad f^{ \pm}(t, x)=f^{ \pm}(0, x \mp t) .
$$

Remark 3.1 The (formally) second-order centered discretization,

$$
M_{\Delta x}\left\{\partial_{x} u\right\}(x) \simeq \frac{1}{2 \Delta x}(u(x+\Delta x)-u(x-\Delta x)),
$$


rewrites in both a "conservative form", involving the numerical flux,

$$
F(a, b)=\frac{a+b}{2}, \quad a=u(x), \quad b=u(x+\Delta x),
$$

and an "average form", i.e.,

$$
\frac{\delta[u]\left(x+\frac{\Delta x}{2}\right)+\delta[u]\left(x-\frac{\Delta x}{2}\right)}{2}, \quad \delta[u]\left(x+\frac{\Delta x}{2}\right)=\frac{u(x+\Delta x)-u(x)}{\Delta x} .
$$

Expression (2.3) of Kirchhoff's solution contains a Laplacian, because even if a (semi-discrete) centered discretization exactly preserves the $L^{2}$ norm,

$$
\begin{aligned}
\frac{d}{d t} \sum_{j} \Delta x\left|u\left(t, x_{j}\right)\right|^{2} & =-\sum_{j} u\left(t, x_{j}\right)\left(\delta[u]\left(t, x_{j}+\frac{\Delta x}{2}\right)+\delta[u]\left(t, x_{j}-\frac{\Delta x}{2}\right)\right) \\
& =-2 \sum_{j} \mu[u]\left(t, x_{j}+\frac{\Delta x}{2}\right) \cdot \delta[u]\left(t, x_{j}+\frac{\Delta x}{2}\right)=0,
\end{aligned}
$$

where $\mu[u]\left(x_{j}+\frac{\Delta x}{2}\right)=\frac{1}{2}\left(u\left(x_{j}\right)+u\left(x_{j}+\Delta x\right)\right)$ is the arithmetic average, its discrete counterpart is unstable except if an implicit time-integrator is used.

\section{Practical computation of numerical fluxes}

Both formulations of (2.3) and (3.1)-(3.2) are "semi-discrete" in the sense that the needed spatial discretization of operators $\nabla$, div and $\Delta$ aren't specified yet.

4.1 Reduction of spherical integrals

For pointwise discrete data on a 2D uniform Cartesian grid, spherical means read,

$$
\begin{aligned}
\forall R>0, \quad M_{R}\{\phi\}(\mathbf{x}) & =\frac{\bar{\phi}}{2 \pi R} \int_{\theta_{1}}^{\theta_{2}} \int_{0}^{R} \frac{r}{\sqrt{R^{2}-r^{2}}} d r, d \theta \\
& =\bar{\phi}\left(\frac{\theta_{2}-\theta_{1}}{2 \pi R}\right)\left[-\sqrt{R^{2}-r^{2}}\right]_{0}^{R} \\
& =\bar{\phi}\left(\frac{\theta_{2}-\theta_{1}}{2 \pi}\right), \quad \bar{\phi} \text { a constant. }
\end{aligned}
$$

where we used that $\phi$ is piecewise constant. For instance, the angular sectors, of width $\frac{\pi}{2}$, where (nodal) gradients are assumed to be constants are displayed in Fig. 4.2: let $\mathbf{x}=\left(x_{i}, y_{j}\right)$ be any (square) cell's center,

$$
M_{\Delta t}\{\phi\}\left(x_{i}, y_{j}\right) \simeq \frac{\pi / 2}{2 \pi}\left(\phi_{i-\frac{1}{2}, j-\frac{1}{2}}+\phi_{i+\frac{1}{2}, j-\frac{1}{2}}+\phi_{i-\frac{1}{2}, j+\frac{1}{2}}+\phi_{i+\frac{1}{2}, j+\frac{1}{2}}\right),
$$

so that spherical means in (2.3) can be reliably approximated by arithmetic averages. 
4.2 Centered part of fluxes

The derivation of the "central part" in (3.1)-(3.2) is illustrated in Fig. 4.1.

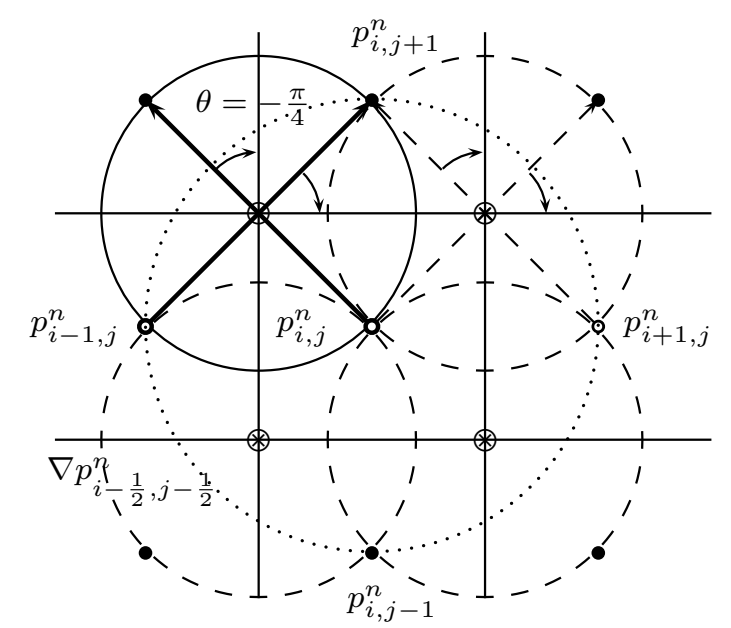

Fig. 4.1 Gradients at each node surrounding a given cell centered at $x_{i}, y_{j}$ : a $-\frac{\pi}{4}$-rotation acts on finite-differences computed along diagonal directions

Basically, around each control cell of size $\Delta x \times \Delta y=\Delta x^{2}$ (the simplest, uniform, Cartesian grid) centered in $x_{i}, y_{j}, i, j \in \mathbb{Z}$, there are 4 nodes $x_{i \pm \frac{1}{2}}, y_{j \pm \frac{1}{2}}$, where it is customary to compute odd-order (partial) derivatives. Hereafter, for a given variable $\mathbb{R}^{2} \rightarrow \mathbb{R}$, say $p\left(t^{n}, x, y\right)$, we shall denote $\nabla p_{i \pm \frac{1}{2}, j \pm \frac{1}{2}}^{n}$ the corresponding approximation of its gradient at each node $x_{i \pm \frac{1}{2}}, y_{j \pm \frac{1}{2}}$. Define $R_{\theta}$ as also the $2 \times 2$ rotation matrix, given by

$$
R_{\theta}=\left(\begin{array}{cc}
\cos \theta & \sin \theta \\
-\sin \theta & \cos \theta
\end{array}\right)
$$

Our centered fluxes, relying on a discrete set of values $p_{i, j}^{n}$, read:

$$
R_{\frac{\pi}{4}} \nabla p_{i-\frac{1}{2}, j+\frac{1}{2}}^{n}=\frac{1}{\Delta x \sqrt{2}}\left(\begin{array}{l}
p_{i, j+1}^{n}-p_{i-1, j}^{n} \\
p_{i-1, j+1}^{n}-p_{i, j}^{n}
\end{array}\right)
$$

Since $\left(R_{\frac{\pi}{4}}\right)^{-1}=R_{-\frac{\pi}{4}}=\left(R_{\frac{\pi}{4}}\right)^{T}$, yields easily that,

$$
\nabla p_{i-\frac{1}{2}, j+\frac{1}{2}}^{n}=\frac{1}{\Delta x}\left(\begin{array}{l}
\frac{p_{i, j}^{n}+p_{i, j+1}^{n}}{2}-\frac{p_{i-1, j}^{n}+p_{i-1, j+1}^{n}}{p_{i-1, j+1}^{n}+p_{i, j+1}^{n}}-\frac{p_{i-1, j}^{n}+p_{i, j}^{n}}{2}
\end{array}\right)
$$

that is to say, a vector of centered finite-differences like (3.3). 
Lemma 4.1 Let $x_{i}, y_{j}$ be any point in the $2 D$ uniform Cartesian grid, then

$$
\begin{aligned}
M_{\Delta t}\{\operatorname{div} \mathbf{u}\}\left(x_{i}, y_{j}\right)=\frac{1}{4 \Delta x} & {\left[\left(\frac{u_{i+1, j+1}+u_{i+1, j}}{2}-\frac{u_{i-1, j+1}+u_{i-1, j}}{2}\right)\right.} \\
& +\left(\frac{u_{i+1, j-1}+u_{i+1, j}}{2}-\frac{u_{i-1, j-1}+u_{i-1, j}}{2}\right) \\
& +\left(\frac{v_{i-1, j+1}+v_{i, j+1}}{2}-\frac{v_{i-1, j-1}+v_{i, j-1}}{2}\right) \\
& \left.+\left(\frac{v_{i+1, j+1}+v_{i, j+1}}{2}-\frac{v_{i+1, j-1}+v_{i, j-1}}{2}\right)\right], \\
M_{\Delta t}\{\nabla p\}\left(x_{i}, y_{j}\right)=\frac{1}{4 \Delta x} & \left(\begin{array}{c}
\frac{p_{i+1, j}+p_{i+1, j+1}}{2}-\frac{p_{i-1, j}+p_{i-1, j+1}}{2} \\
+\frac{p_{i+1, j}+p_{i+1, j-1}}{2}-\frac{p_{i-1, j}+p_{i-1, j-1}}{2} \\
\frac{p_{i-1, j+1}+p_{i, j+1}}{2}-\frac{p_{i-1, j-1}+p_{i, j-1}}{2} \\
+\frac{p_{i+1, j+1}+p_{i, j+1}}{2}-\frac{p_{i+1, j-1}+p_{i, j-1}}{2}
\end{array}\right),
\end{aligned}
$$

follow by assuming that nodal gradients hold in each $\frac{\pi}{2}$ angular sector.

Proof Both formulas follow from previous calculations (involving $R_{\frac{\pi}{4}}$ ) by taking advantage of simple cancellations in each expression.

Remark 4.1 Formula (2.3) is convenient for a wave equation (1.4) in which the propagation speed $c \equiv 1$ everywhere, so that the choice $\Delta x=\Delta y$ is very natural; however, one may think about using (2.3), hence (3.1)-(3.2) in a more general context for which $\Delta x \neq \Delta y$. Accordingly, the angle $\theta$ is defined through,

$$
\tan \theta=\frac{\Delta y}{\Delta x}, \quad 0<\theta<\frac{\pi}{2},
$$

so that the numerical expression of the gradient is slightly more complex,

$$
\begin{aligned}
& \nabla p_{i-\frac{1}{2}, j+\frac{1}{2}}^{n}=\left(\begin{array}{l}
\frac{\left(p_{i, j+1}^{n} \cos \theta+p_{i, j}^{n} \sin \theta\right)-\left(p_{i-1, j}^{n} \cos \theta+p_{i-1, j+1}^{n} \sin \theta\right)}{\sqrt{\Delta x^{2}+\Delta y^{2}}} \\
\frac{\left(p_{i-1, j+1}^{n} \cos \theta+p_{i, j+1}^{n} \sin \theta\right)-\left(p_{i, j}^{n} \cos \theta+p_{i-1, j}^{n} \sin \theta\right)}{\sqrt{\Delta x^{2}+\Delta y^{2}}}
\end{array}\right) \\
& =\left(\begin{array}{l}
\left.\frac{p_{i, j+1}^{n}(1+\cos 2 \theta)+p_{i, j}^{n} \sin 2 \theta}{2 \Delta x}-\frac{p_{i-1, j+1}^{n} \sin 2 \theta+p_{i-1, j}^{n}(1+\cos 2 \theta)}{2 \Delta x}\right) \\
\frac{p_{i, j+1}^{n}(1-\cos 2 \theta)+p_{i-1, j+1}^{n} \sin 2 \theta}{2 \Delta y}-\frac{p_{i, j}^{n} \sin 2 \theta+p_{i-1, j}^{n}(1-\cos 2 \theta)}{2 \Delta y}
\end{array}\right)
\end{aligned}
$$


where the following relations are used:

$$
\frac{1}{\sqrt{\Delta x^{2}+\Delta y^{2}}}=\sqrt{\frac{\cos ^{2} \theta}{\Delta x^{2}\left(\cos ^{2} \theta+\sin ^{2} \theta\right)}}=\frac{\cos \theta}{\Delta x}=\frac{\sin \theta}{\Delta y} .
$$

\subsection{Diffusive part of fluxes}

In order to compute an approximate Laplacian operator at the center $x_{i}, y_{j}$ of each cell, we shall apply the same method: starting from nodal gradient values, diagonal finite-differences are formed, so that second-order derivatives are retrieved by applying $R_{-\frac{\pi}{4}}$. More precisely, let $\left(p_{x}\right)_{i-\frac{1}{2}, j-\frac{1}{2}}$ stand for the

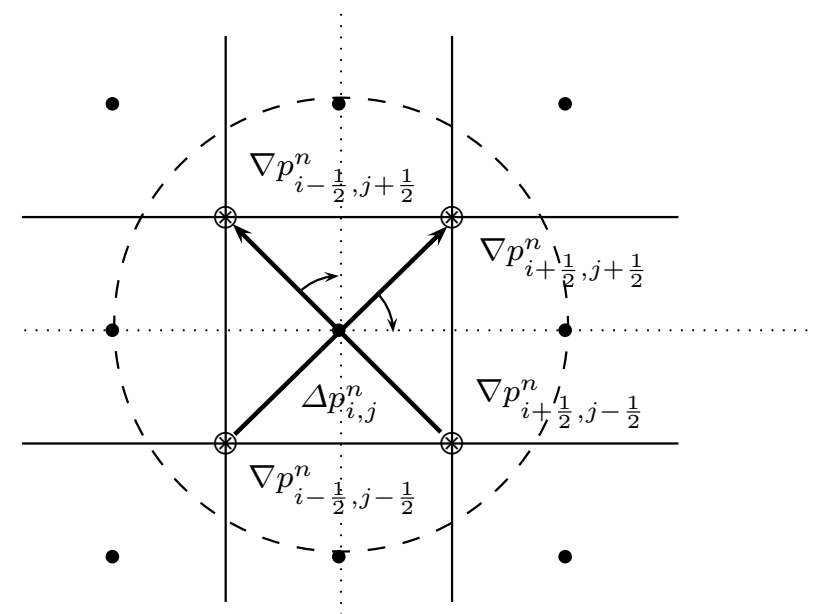

Fig. 4.2 Laplacian term at $x_{i}, y_{j}$ : a $-\frac{\pi}{4}$-rotation acts on nodal gradients.

first component of the gradient vector $\nabla p_{i-\frac{1}{2}, j-\frac{1}{2}}$ in $x_{i-\frac{1}{2}}, y_{j-\frac{1}{2}}$, as computed in the previous section. Then, with similar notation, yields,

$$
R_{\frac{\pi}{4}}\left(\begin{array}{c}
\left(p_{x x}\right)_{i, j} \\
\left(p_{x y}\right)_{i, j}
\end{array}\right)=\frac{1}{\Delta x \sqrt{2}}\left(\begin{array}{c}
\left(p_{x}\right)_{i+\frac{1}{2}, j+\frac{1}{2}}-\left(p_{x}\right)_{i-\frac{1}{2}, j-\frac{1}{2}} \\
\left(p_{x}\right)_{i-\frac{1}{2}, j+\frac{1}{2}}-\left(p_{x}\right)_{i+\frac{1}{2}, j-\frac{1}{2}}
\end{array}\right)
$$

together with,

$$
R_{\frac{\pi}{4}}\left(\begin{array}{c}
\left(p_{y x}\right)_{i, j} \\
\left(p_{y y}\right)_{i, j}
\end{array}\right)=\frac{1}{\Delta x \sqrt{2}}\left(\begin{array}{c}
\left(p_{y}\right)_{i+\frac{1}{2}, j+\frac{1}{2}}-\left(p_{y}\right)_{i-\frac{1}{2}, j-\frac{1}{2}} \\
\left(p_{y}\right)_{i-\frac{1}{2}, j+\frac{1}{2}}-\left(p_{y}\right)_{i+\frac{1}{2}, j-\frac{1}{2}}
\end{array}\right)
$$


Lemma 4.2 Let $x_{i}, y_{j}$ be any point in the $2 D$ uniform Cartesian grid, then

$$
\begin{aligned}
&(\Delta p)_{i, j}= \frac{1}{4}\left(\frac{p_{i+1, j+1}^{n}-2 p_{i, j+1}^{n}+p_{i-1, j+1}^{n}}{\Delta x^{2}}\right. \\
&+2 \frac{p_{i+1, j}^{n}-2 p_{i, j}^{n}+p_{i-1, j}^{n}}{\Delta x^{2}}\left.+\frac{p_{i+1, j-1}^{n}-2 p_{i, j-1}^{n}+p_{i-1, j-1}^{n}}{\Delta x^{2}}\right) \\
&+\frac{1}{4}\left(\frac{p_{i+1, j+1}^{n}-2 p_{i+1, j}^{n}+p_{i+1, j-1}^{n}}{\Delta x^{2}}\right. \\
&+2 \frac{p_{i, j+1}^{n}-2 p_{i, j}^{n}+p_{i, j-1}^{n}}{\Delta x^{2}} \\
&\left.+\frac{p_{i-1, j+1}^{n}-2 p_{i-1, j}^{n}+p_{i-1, j-1}^{n}}{\Delta x^{2}}\right)
\end{aligned}
$$

Moreover, the numerical Hessian is a symmetric $2 \times 2$ matrix.

Proof According to former definitions and Lemma 4.1,

$$
\begin{aligned}
& \left(p_{x x}\right)_{i, j}=\frac{1}{\Delta x}\left[\frac{\left(p_{x}\right)_{i+\frac{1}{2}, j+\frac{1}{2}}+\left(p_{x}\right)_{i+\frac{1}{2}, j-\frac{1}{2}}}{2}-\frac{\left(p_{x}\right)_{i-\frac{1}{2}, j-\frac{1}{2}}+\left(p_{x}\right)_{i-\frac{1}{2}, j+\frac{1}{2}}}{2}\right], \\
& \left(p_{y y}\right)_{i, j}=\frac{1}{\Delta x}\left[\frac{\left(p_{y}\right)_{i+\frac{1}{2}, j+\frac{1}{2}}+\left(p_{y}\right)_{i-\frac{1}{2}, j+\frac{1}{2}}}{2}-\frac{\left(p_{y}\right)_{i+\frac{1}{2}, j-\frac{1}{2}}+\left(p_{y}\right)_{i-\frac{1}{2}, j-\frac{1}{2}}}{2}\right],
\end{aligned}
$$

and it suffices to insert values from Lemma 4.1 to reach (4.4). Oppositely,

$$
\begin{aligned}
\left(p_{x y}\right)_{i, j}=\frac{1}{\Delta x}\left[\frac{\left(p_{x}\right)_{i+\frac{1}{2}, j+\frac{1}{2}}+\left(p_{x}\right)_{i-\frac{1}{2}, j+\frac{1}{2}}}{2}-\frac{\left(p_{x}\right)_{i+\frac{1}{2}, j-\frac{1}{2}}+\left(p_{x}\right)_{i-\frac{1}{2}, j-\frac{1}{2}}}{2}\right] \\
=\frac{1}{4 \Delta x^{2}}\left[\left(\left[p_{i+1, j+1}+p_{i+1, j}\right)-\left(\left[p_{i, j+1}+p_{i, j}\right)\right.\right.\right. \\
+\left(p_{i, j+1}+p_{i, j}\right)-\left(p_{i-1, j+1}+p_{i-1, j}\right) \\
\quad-\left(p_{i+1, j}+p_{i+1, j-1}\right)+\left(p_{i, j}+p_{i, j-1}\right) \\
\left.\quad-\left(p_{i, j}+p_{i, j-1}\right)+\left(p_{i-1, j}+p_{i-1, j-1}\right)\right] \\
=\frac{1}{\Delta x}\left[\frac{\left.\left(p_{y}\right)_{i+\frac{1}{2}, j+\frac{1}{2}}+\left(p_{y}\right)_{i+\frac{1}{2}, j-\frac{1}{2}}-\frac{\left(p_{y}\right)_{i-\frac{1}{2}, j+\frac{1}{2}}+\left(p_{y}\right)_{i-\frac{1}{2}, j-\frac{1}{2}}}{2}\right]}{2}\right. \\
=\left(p_{y x}\right)_{i, j},
\end{aligned}
$$

because the "boxed values" correspond to $\left(p_{y}\right)_{i+\frac{1}{2}, j+\frac{1}{2}}-\left(p_{y}\right)_{i-\frac{1}{2}, j+\frac{1}{2}}$. 
4.4 Conservative 9-point Kirchhoff-based scheme

Lemmas 4.1 and 4.2 furnish necessary ingredients in order to set up a fully discrete version of (3.1)-(3.2) on a Cartesian grid $\Delta x=\Delta y$. To alleviate its presentation, we borrow notation from [20,21]: for any $\mathbf{x}=(x, y) \in \mathbb{R}^{2}$,

$$
\begin{aligned}
& \mu_{x}[p](\mathbf{x})=\frac{1}{2}\left(p\left(x+\frac{\Delta x}{2}, y\right)+p\left(x-\frac{\Delta x}{2}, y\right)\right), \\
& \mu_{y}[p](\mathbf{x})=\frac{1}{2}\left(p\left(x, y+\frac{\Delta x}{2}\right)+p\left(x, y-\frac{\Delta x}{2}\right)\right), \\
& \delta_{x}[p](\mathbf{x})=\frac{1}{\Delta x}\left(p\left(x+\frac{\Delta x}{2}, y\right)-p\left(x-\frac{\Delta x}{2}, y\right)\right), \\
& \delta_{y}[p](\mathbf{x})=\frac{1}{\Delta x}\left(p\left(x, y+\frac{\Delta x}{2}\right)-p\left(x, y-\frac{\Delta x}{2}\right)\right) .
\end{aligned}
$$

Thus $\delta_{x}$ and $\mu_{x}$ commute to produce centered derivatives (see Remark 3.1),

$$
\forall \mathbf{x} \in \mathbb{R}^{2}, \quad \mu_{x}\left[\delta_{x}[p]\right](\mathbf{x})=\frac{p(x+\Delta x, y)-p(x-\Delta x, y)}{2 \Delta x}=\delta_{x}\left[\mu_{x}[p]\right](\mathbf{x}) .
$$

Moreover, assuming that (4.4) holds uniformly in the disks centered in $\mathbf{x}_{i, j}$,

$$
\begin{aligned}
\int_{0}^{\Delta t} \tau M_{\tau}\{\Delta p\}\left(t^{n}, \mathbf{x}_{i, j}\right) d \tau & \simeq \frac{\Delta t^{2}}{2}(\Delta p)_{i, j}^{n} \\
& =\frac{\Delta t^{2}}{2}\left(\left(\delta_{x} \mu_{y}\right)^{2}+\left(\delta_{y} \mu_{x}\right)^{2}\right)[p]\left(t^{n}, x_{i}, y_{j}\right) .
\end{aligned}
$$

Lemma 4.3 On a Cartesian grid where $\Delta x=\Delta y$ and $\lambda=\frac{\Delta t}{\Delta x}$, the scheme (3.1)-(3.2) rewrites as a method of lines: for $t>0$ and $\mathbf{x}=(i \Delta x, j \Delta y)$,

$$
\begin{aligned}
\frac{d}{d t}\left(\begin{array}{c}
p(t, \mathbf{x}) \\
u(t, \mathbf{x}) \\
v(t, \mathbf{x})
\end{array}\right)+ & \left(\begin{array}{c}
\mu_{x}\left[\delta_{x}\left[\mu_{y}^{2}[u]\right]\right](t, \mathbf{x}) \\
\mu_{x}\left[\delta_{x}\left[\mu_{y}^{2}[p]\right]\right](t, \mathbf{x}) \\
0 \\
0
\end{array}\right)+\left(\begin{array}{c}
\mu_{y}\left[\delta_{y}\left[\mu_{x}^{2}[v]\right]\right](t, \mathbf{x}) \\
0 \\
\mu_{y}\left[\delta_{y}\left[\mu_{x}^{2}[p]\right]\right](t, \mathbf{x})
\end{array}\right) \\
& =\frac{\lambda \Delta x}{2}\left(\begin{array}{c}
\left(\delta_{x} \mu_{y}\right)^{2}[p](t, \mathbf{x})+\left(\delta_{y} \mu_{x}\right)^{2}[p](t, \mathbf{x}) \\
\left(\delta_{x} \mu_{y}\right)^{2}[u](t, \mathbf{x})+\left(\delta_{y} \mu_{x}\right)^{2}[u](t, \mathbf{x}) \\
\left(\delta_{x} \mu_{y}\right)^{2}[v](t, \mathbf{x})+\left(\delta_{y} \mu_{x}\right)^{2}[v](t, \mathbf{x})
\end{array}\right) .
\end{aligned}
$$

Since both $\mu_{x}, \delta_{x}$ and $\mu_{y}, \delta_{y}$ commute with each other, the resulting differential system (4.5) is conservative with (edge-based) numerical fluxes,

$$
\begin{aligned}
\forall t>0, \quad F_{i-\frac{1}{2}, j}(t)= & \left(\begin{array}{r}
\left(\mu_{x}\left[\mu_{y}^{2}[u]\right]-\frac{\lambda \Delta x}{2} \delta_{x}\left[\mu_{y}^{2}[p]\right]\right)\left(t, x_{i-\frac{1}{2}}, y_{j}\right) \\
\left.\left(\mu_{x}\left[\mu_{y}^{2}[p]\right]\right]-\frac{\lambda \Delta x}{2} \delta_{x}\left[\mu_{y}^{2}[u]\right]\right)\left(t, x_{i-\frac{1}{2}}, y_{j}\right) \\
-\frac{\lambda \Delta x}{2} \delta_{x}\left[\mu_{y}^{2}[v]\right]\left(t, x_{i-\frac{1}{2}}, y_{j}\right)
\end{array}\right), \\
G_{i, j-\frac{1}{2}}(t) & =\left(\begin{array}{r}
\left(\mu_{y}\left[\mu_{x}^{2}[v]\right]-\frac{\lambda \Delta x}{2} \delta_{y}\left[\mu_{x}^{2}[p]\right]\right)\left(t, x_{i}, y_{j-\frac{1}{2}}\right) \\
-\frac{\lambda \Delta x}{2} \delta_{y}\left[\mu_{x}^{2}[u]\right]\left(t, x_{i}, y_{j-\frac{1}{2}}\right) \\
\left(\mu_{y}\left[\mu_{x}^{2}[p]\right]-\frac{\lambda \Delta x}{2} \delta_{y}\left[\mu_{x}^{2}[v]\right]\right)\left(t, x_{i}, y_{j-\frac{1}{2}}\right)
\end{array}\right) .
\end{aligned}
$$


Proof To establish (4.5), one rewrites the contents of Lemmas 4.1 and 4.2 with the (arithmetic) averaging $\mu$. and finite-differencing $\delta$. operators.

Remark 4.2 Expression (4.5) can be modified according to [18, eqn. (5)] because,

$$
\left[\mu_{x}\right]^{2}=I d+\frac{\Delta x^{2}}{4}\left[\delta_{x}\right]^{2}, \quad\left[\mu_{y}\right]^{2}=I d+\frac{\Delta x^{2}}{4}\left[\delta_{y}\right]^{2},
$$

in the sense of operators. It then appears that (4.5) differs from the (isotropic, vorticity-preserving) scheme given by $\alpha_{1}=\alpha_{2}=\phi_{1}=\phi_{2}=\frac{1}{4}$ in [18, Part I] only for two viscous terms, which moreover cancel if the numerical solution is curl-free.

\section{Vorticity and $H^{s}$ (semi-discrete) stability result}

We prove a numerical counterpart of Theorem 1.1, in analogy with [20] and so-called "potential-based schemes"; specifically, (4.5) appears to be identical to a "symmetric Rusanov potential scheme", as written in [20, eqn. (3.12)]. Such an algorithm doesn't preserve the vorticity (5.3) because equations [20, (3.10)-(3.11)] don't yield [20, (3.12)], so that the statement of [20, Theorem 3.2] holds only for the energy estimate, but not for the vorticity preservation.

5.1 Semi-discrete $L^{2}$ and $H^{s}$ stability estimates

There holds:

Theorem 5.1 Assume initial data $p_{0}(\mathbf{x}), \mathbf{u}_{0}(\mathbf{x})$ belong to $H^{s}\left(\mathbb{R}^{2}\right)$ for some $s \in \mathbb{N}_{*}$, then, given any $\Delta x>0$ and $t>0$, the solution of (4.5) satisfies

$$
\Delta x^{2} \sum_{i, j \in \mathbb{Z}^{2}} p\left(t, x_{i}, y_{j}\right)^{2}+\left|\mathbf{u}\left(t, x_{i}, y_{j}\right)\right|^{2} \leq \Delta x^{2} \sum_{i, j \in \mathbb{Z}^{2}} p_{0}\left(x_{i}, y_{j}\right)^{2}+\left|\mathbf{u}_{0}\left(x_{i}, y_{j}\right)\right|^{2}
$$

Moreover, since the scheme (4.5) is shift-invariant, this time-decay holds for any discrete Sobolev norm (of order smaller than $s$ ) as well.

Before attacking the proof of Theorem 5.1, we state an easy lemma:

Lemma 5.1 Let $\mathbf{u}=(u, v): \mathbb{R}^{2} \rightarrow \mathbb{R}^{2}$ be sampled on a Cartesian grid, then

$$
\begin{aligned}
\sum_{i, j \in \mathbb{Z}^{2}} v\left(x_{i}, y_{j}\right) \cdot \mu_{x}\left[\delta_{x}[u]\right]\left(x_{i}, y_{j}\right) & =2 \sum_{i, j \in \mathbb{Z}^{2}} \mu_{x}[v] \cdot \delta_{x}[u]\left(x_{i+\frac{1}{2}}, y_{j}\right), \\
\forall n \in \mathbb{N}, \quad \sum_{i, j \in \mathbb{Z}^{2}}\left(\mu_{x}\right)^{n}[v]\left(x_{i+\left[\frac{n}{2}\right] / 2}, y_{j}\right) & =\sum_{i, j \in \mathbb{Z}^{2}} v\left(x_{i}, y_{j}\right) .
\end{aligned}
$$


Proof The proof of Theorem 5.1 splits into two distinct steps: first, showing that "centered terms" cancel in the summation, and second, that "diffusive terms" bring a negative contribution thanks to Jensen's inequality.

- "centered differences": one multiplies the first line of (4.5) by $p_{i, j}$, sums on both indexes $i, j \in \mathbb{Z}^{2}$, and applies (5.1), results in:

$$
\sum_{j} \sum_{i} 2\left(\mu_{x}[p]\right)_{i+\frac{1}{2}, j} \cdot \delta_{x}\left[\mu_{y}^{2}[u]\right]_{i+\frac{1}{2}, j}+\sum_{i} \sum_{j} 2\left(\mu_{y}[p]_{i, j+\frac{1}{2}} \cdot \delta_{y}\left[\mu_{x}^{2}[v]\right]_{i, j+\frac{1}{2}}\right.
$$

Similarly, one multiplies the second (third) line of (4.5) by $u_{i, j}$ (by $v_{i, j}$ ), sums on both indexes $i, j \in \mathbb{Z}^{2}$, applies (5.1), and adds:

$$
\sum_{j} \sum_{i} 2\left(\mu_{x}[u]\right)_{i+\frac{1}{2}, j} \cdot \delta_{x}\left[\mu_{y}^{2}[p]\right]_{i+\frac{1}{2}, j}+\sum_{i} \sum_{j} 2\left(\mu_{y}[v]_{i, j+\frac{1}{2}} \cdot \delta_{y}\left[\mu_{x}^{2}[p]\right]_{i, j+\frac{1}{2}}\right.
$$

Yet, by applying (5.2), results in that, for instance,

$\sum_{j} \sum_{i} \mu_{x}[p]_{i+\frac{1}{2}, j} \cdot \delta_{x}\left[\mu_{y}^{2}[u]\right]_{i+\frac{1}{2}, j}=\sum_{j} \sum_{i} \mu_{x}\left[\mu_{y}[p]\right]_{i+\frac{1}{2}, j+\frac{1}{2}} \cdot \delta_{x}\left[\mu_{y}[u]\right]_{i+\frac{1}{2}, j+\frac{1}{2}}$,

so that, by adding everything and using commutation of $\mu_{x}, \mu_{y}$,

$$
\begin{gathered}
\sum_{i, j \in \mathbb{Z}^{2}} \mu_{x}\left[\mu_{y}[p+u]\right]_{i+\frac{1}{2}, j+\frac{1}{2}} \cdot \delta_{x}\left[\mu_{y}[u+p]\right]_{i+\frac{1}{2}, j+\frac{1}{2}}=0, \\
\sum_{i, j \in \mathbb{Z}^{2}} \mu_{y}\left[\mu_{x}[p+v]\right]_{i+\frac{1}{2}, j+\frac{1}{2}} \cdot \delta_{y}\left[\mu_{x}[v+p]\right]_{i+\frac{1}{2}, j+\frac{1}{2}}=0 .
\end{gathered}
$$

- "diffusive terms": since diffusive fluxes are decoupled, computations will be shown only for the $p$ variable, the other ones being very similar.

$$
\begin{array}{r}
\sum_{i, j \in \mathbb{Z}^{2}} p_{i, j}\left(\left(\delta_{x} \mu_{y}\right)^{2}[p]+\left(\delta_{y} \mu_{x}\right)^{2}[p]\right)= \\
-\sum_{i, j \in \mathbb{Z}^{2}}\left|\delta_{x}\left[\mu_{y}[p]\right]_{i+\frac{1}{2}, j+\frac{1}{2}}\right|^{2}-\sum_{i, j \in \mathbb{Z}^{2}}\left|\delta_{y}\left[\mu_{x}[p]\right]_{i+\frac{1}{2}, j+\frac{1}{2}}\right|^{2} \leq 0
\end{array}
$$

the negative terms being summable for data belonging to $H^{1}\left(\mathbb{R}^{2}\right)$.

Consequently, the solution of (4.5) satisfies,

$$
\forall t>0, \quad \frac{d}{d t}\left(\Delta x^{2} \sum_{i, j \in \mathbb{Z}^{2}}\left|p\left(t, x_{i}, y_{j}\right)\right|^{2}+\left|\mathbf{u}\left(t, x_{i}, y_{j}\right)\right|^{2}\right) \leq 0 .
$$

Finally, by linearity and shift-invariance of (4.5), this dissipation estimate extends to all discrete derivatives in $x, y$. 
Remark 5.1 The estimate in Theorem 5.1 doesn't hold automatically for a fully discrete method, except for an implicit scheme where the right-hand side of (4.5) is evaluated in $t^{n+1}$ (in such a case, diffusive terms aren't needed and centered derivatives suffice, see [19, eqn. (2.10)]). For a mid-point, CrankNicolson, time-integrator, the $L^{2}$ estimate holds for any $\Delta t>0$ because,

$$
\left(\frac{p_{i, j}^{n+1}+p_{i, j}^{n}}{2}\right) \cdot \frac{p_{i, j}^{n+1}-p_{i, j}^{n}}{\Delta t}=\frac{1}{2} \frac{\left|p_{i, j}^{n+1}\right|^{2}-\left|p_{i, j}^{n}\right|^{2}}{\Delta t},
$$

and the right-hand side behaves as in the previous proof. However, for an explicit time-marching scheme, the Jensen's inequality implies that,

$$
\frac{1}{2} \frac{\left|p_{i, j}^{n+1}\right|^{2}-\left|p_{i, j}^{n}\right|^{2}}{\Delta t}=\frac{\frac{1}{2}\left(\left|p_{i, j}^{n+1}\right|^{2}+\left|p_{i, j}^{n}\right|^{2}\right)-\left|p_{i, j}^{n}\right|^{2}}{\Delta t} \geq \frac{p_{i, j}^{n} \cdot p_{i, j}^{n+1}-\left|p_{i, j}^{n}\right|^{2}}{\Delta t},
$$

so, in general, the $L^{2}$ norm may increase. A Fourier analysis, see e.g. [14,22, 21 , is probably needed in order to derive an explicit sufficient CFL restriction.

\subsection{Nodal vorticity and irrotationality-preservation}

Part of the results in $[19,20]$ can be stated as: given either symmetric or diagonal potential-based scheme for (1.1), there is a consistent approximation of vorticity which is kept constant along the flow of its "method of lines". Accordingly, following e.g. [21], we define

$$
\forall \mathbf{x}=(i \Delta x, j \Delta x), \quad \omega\left(t, x_{i+\frac{1}{2}}, y_{j+\frac{1}{2}}\right)=\mu_{x}\left[\delta_{y}[u]\right]-\mu_{y}\left[\delta_{x}[v]\right],
$$

as a vertex-based (or nodal) approximate vorticity for the solution of (4.5).

Corollary 5.1 Given any solution of (4.5), its vorticity (5.3) satisfies,

$$
\forall t>0, \quad \frac{d \omega_{i+\frac{1}{2}, j+\frac{1}{2}}}{d t}=\frac{\lambda \Delta x}{2}(\Delta \omega)_{i+\frac{1}{2}, j+\frac{1}{2}},
$$

where the discrete Laplacian is given by (4.4). Accordingly, initial data $\mathbf{u}_{0}(\mathbf{x})$ endowed with an harmonic vorticity, $(\Delta \omega)_{i+\frac{1}{2}, j+\frac{1}{2}} \equiv 0$, is preserved, whereas in general,

$$
\forall t>0, \quad \frac{d}{d t}\left(\sum_{i, j} \Delta x^{2}\left|\omega_{i+\frac{1}{2}, j+\frac{1}{2}}(t)\right|^{2}\right) \leq 0 .
$$

Proof It consists in inserting the expression (5.3) into (4.5), and exploiting commutation properties of discrete operators $\delta ., \mu$.

- First, centered derivatives in both $\frac{d u_{i, j}}{d t}$ and $\frac{d v_{i, j}}{d t}$ cancel:

$$
\begin{aligned}
\left(\mu_{x} \delta_{y}\right)\left[\left(\mu_{x} \delta_{x}\right)\left[\mu_{y}^{2}[p]\right]\right]-\left(\mu_{y} \delta_{x}\right)\left[\left(\mu_{y} \delta_{y}\right)\left[\mu_{x}^{2}[p]\right]\right] & = \\
\left(\delta_{y} \mu_{x}^{2}\right)\left[\left(\delta_{x} \mu_{y}^{2}\right)[p]\right]-\left(\delta_{x} \mu_{y}^{2}\right)\left[\left(\delta_{y} \mu_{x}^{2}\right)[p]\right] & =0
\end{aligned}
$$


- Then, diffusive terms commute with (5.3) in order to produce:

$$
\begin{array}{r}
\left(\mu_{x} \delta_{y}\right)\left[\left(\delta_{x} \mu_{y}\right)^{2}+\left(\delta_{y} \mu_{x}\right)^{2}\right][u]-\left(\mu_{y} \delta_{x}\right)\left[\left(\delta_{x} \mu_{y}\right)^{2}+\left(\delta_{y} \mu_{x}\right)^{2}\right][v] \\
=\left[\left(\delta_{x} \mu_{y}\right)^{2}+\left(\delta_{y} \mu_{x}\right)^{2}\right]\left[\left(\mu_{x} \delta_{y}\right)[u]-\left(\mu_{y} \delta_{x}\right)[v]\right] \\
=\left[\left(\delta_{x} \mu_{y}\right)^{2}+\left(\delta_{y} \mu_{x}\right)^{2}\right][\omega] .
\end{array}
$$

To get (5.5), one mimics the "diffusive terms" calculation already done in the proof of Theorem 5.1, which implies that all the discrete $H^{s}$ norms decrease.

The "heat equation" on $\omega$, derived in Corollary 5.1, isn't something as bad as it may seem: indeed, in order to set up the "Kirchhoff-based scheme" (2.3) for (1.1), we had previously to assume that $\mathbf{u}$ was the curl-free component of $\mathbf{U}$. Accordingly, getting that any numerical "rot u" dissipates like (5.4) appears to be globally consistent with this aforementioned framework.

\section{Numerical assessments}

In this section we propose numerical results for the scheme (4.5).

\subsection{Analytic solution and convergence}

The practical order of convergence, in $\Omega=[0,2]^{2}$, is checked on the following exact solution,

$$
\left\{\begin{array}{l}
p(t, x, y)=-\sqrt{2} \pi \sin (\sqrt{2} \pi t) \cos (\pi x) \cos (\pi y) \\
u_{1}(t, x, y)=\pi \cos (\sqrt{2} \pi t) \sin (\pi x) \cos (\pi y) \\
u_{2}(t, x, y)=\pi \cos (\sqrt{2} \pi t) \cos (\pi x) \sin (\pi y)
\end{array}\right.
$$

Table 6.1 displays the expected convergence order in both the $L^{1}$ and $L^{2}$

\begin{tabular}{|c|c|c|c|c|}
\hline \# cells & \multicolumn{2}{|c|}{$L^{1}$ error } & \multicolumn{2}{c|}{$L^{2}$ error } \\
& error & order & error & order \\
\hline$n=20$ & $4.4 E^{-1}$ & - & $1.6 E^{-1}$ & - \\
\hline$n=40$ & $1.1 E^{-1}$ & 2 & $4.0 E^{-2}$ & 2 \\
\hline$n=80$ & $2.8 E^{-2}$ & 1.98 & $1.0 E^{-2}$ & 2 \\
\hline$n=160$ & $7.0 E^{-3}$ & 2 & $2.5 E^{-3}$ & 2 \\
\hline$n=320$ & $1.75 E^{-3}$ & 1.99 & $6.3 E^{-4}$ & 1.99 \\
\hline
\end{tabular}

Table 6.1 Measured orders of convergence for a smooth solution.

norms. The scheme can be interpreted as a extension of the Lax-Wendroff scheme which is a second order scheme. 
6.2 Numerical acoustic wave propagation

Consider a domain $\Omega=[0,3]^{2}$, a computational mesh $300 \times 300$ in order to compute until a final time of $T_{f}=1$ the numerical solution emerging from (smooth, discontinuous or Dirac) initial data:

test $1: p(t=0, x, y)=e^{-10\left((x-3)^{2}+(y-3)^{2}\right)}, u_{1}(t=0, \cdot)=u_{2}(t=0, \cdot) \equiv 0$.

Fig. 6.1 shows that the scheme is efficient, displays little dispersion effects
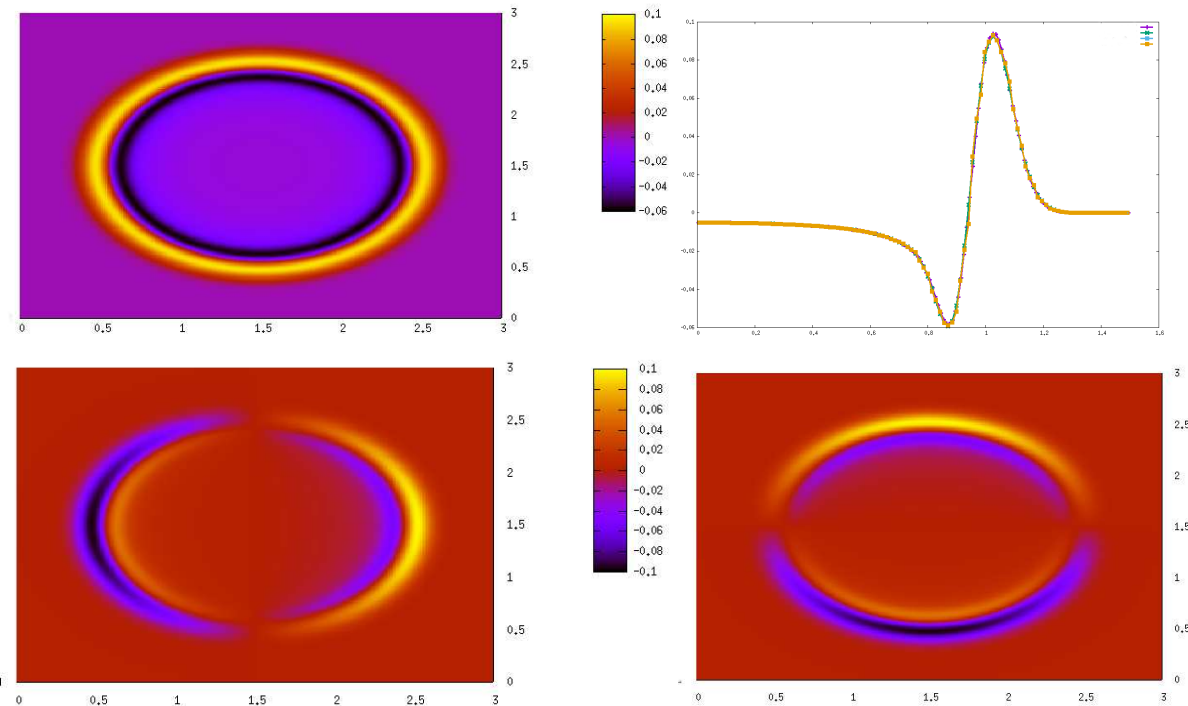

Fig. 6.1 Top: final pressure (2D and radial cuts). Bottom: horizontal and vertical velocities. On top, right, four pressure "radial cuts" are superimposed in order to show isotropy.

(on radial plots), and captures a typical second-order accurate (classical) solution. In particular, Fig. 6.2 displays the "mesh imprinting" for (4.5) by showing radial cuts in the numerical pressure for the angles $0, \frac{\pi}{8}, \frac{\pi}{4}, \frac{3 \pi}{8}, \frac{\pi}{2}$.

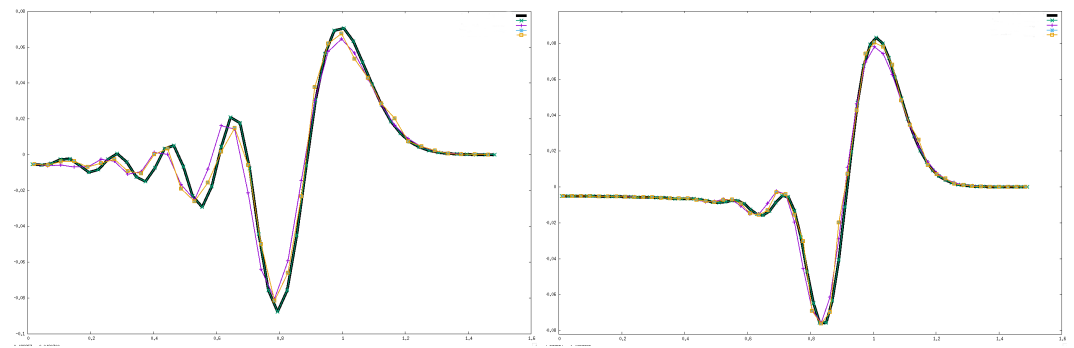

Fig. 6.2 Pressure "radial cuts" for $100 \times 100$ (left) and $150 \times 150$ (right) grids. 
test $2: p(t=0, x, y)=\chi(x, y)_{B}, u_{1}(t=0, x, y)=u_{2}(t=0, x, y) \equiv 0$, with

$$
B:=B\left(x_{0}, y_{0}, r\right)=\left\{(x, y) /\left|x-x_{0}\right|^{2}+\left|y-y_{0}\right|^{2}<r^{2}\right\}, \quad x_{0}=y_{0}=3,
$$

On Fig. 6.3, dynamics are still correctly rendered, but slightly anisotropic
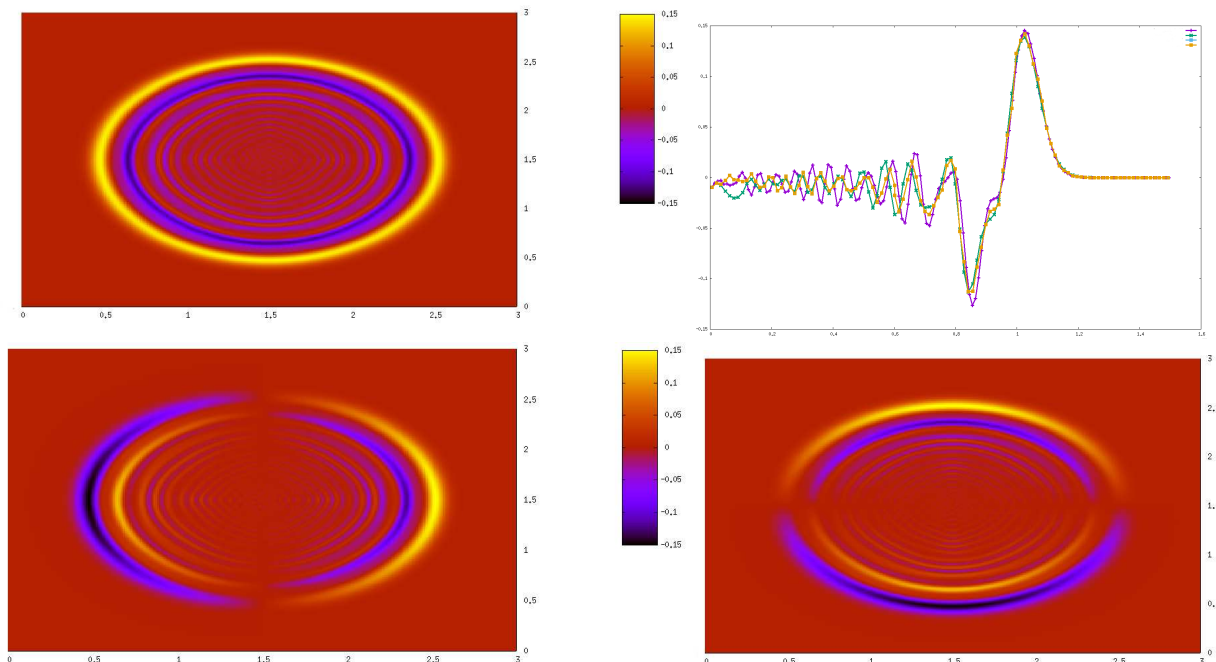

Fig. 6.3 Top: final pressure (2D and radial cuts). Bottom: horizontal and vertical velocities. Top, right: four pressure "radial cuts" are superimposed and reveal a small lack of isotropy.

oscillations appear, in agreement with classical results on second order schemes, in which (initial) sharp fronts may produce oscillations.

test $3: p(t=0, x, y) \simeq \delta(x-3, y-3), u_{1}(t=0, x, y)=u_{2}(t=0, x, y) \equiv 0$. Fig. 6.4
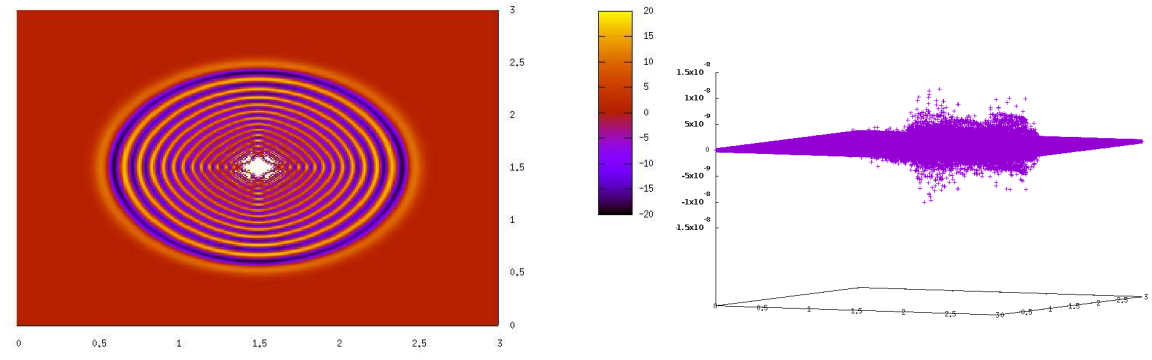

Fig. 6.4 Final pressure (left) and numerical vorticity (right) of order $10^{-8}$ for test 3 .

confirms that the scheme produces spurious modes when it is initialized with an approximation of the Dirac measure. However, since the initial vorticity is equal to zero, it stays so (at machine accuracy) for all these 
three benchmarks. With Dirac data and for $\theta$ the Heaviside function, the exact solution reads,

$$
p(t, x, y)=\frac{1}{2 \pi} \frac{\partial}{\partial t}\left(\frac{\theta(t-r)}{\sqrt{\left|t^{2}-r^{2}\right|}}\right), \quad r^{2}=(x-3)^{2}+(y-3)^{2} .
$$

Previous cases show that irrotational data is preserved, in agreement with

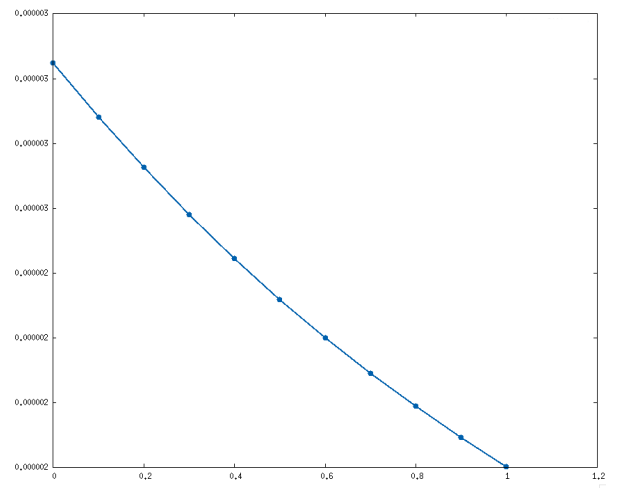

Fig. 6.5 Evolution in time of the $L^{2}$ norm of the vorticity for test 4 .

Corollary 5.1, which also states that a non-zero initial vorticity is dissipated in the $L^{2}$ norm. Accordingly, for a final time $T_{f}=1$, a domain $\Omega=[0,4]^{2}$ gridded by $200 \times 200$ points,

$$
\begin{aligned}
p(t=0, x, y) & =e^{-10\left((x-3)^{2}+(y-3)^{2}\right)} \\
u_{1}(t=0, x, y) & =0.01(y-3) p(t=0, x, y), \\
u_{2}(t=0, x, y) & =-0.01(x-3) p(t=0, x, y) \\
\omega(x, y)=\partial_{y} u_{1}-\partial_{x} u_{2} & =0.2 p(t=0, x, y)\left(\frac{1}{10}-\left((x-3)^{2}+(y-3)^{2}\right)\right) .
\end{aligned}
$$

Fig 6.5 shows the corresponding time-evolution of vorticity in the $L^{2}$ norm.

Remark 6.1 Our scheme (4.5) is identical to the one in [20, eqn. (3.12)], despite it dissipates the vorticity (5.3) according to the "heat equation" (5.4), as illustrated in Fig. 6.5. This isn't in contradiction with the numerical tests preformed in both $[19, \S 3.1-2]$ and $[20, \S 4.2]$ because all the considered initial data for (1.1) are chosen curl-free, and such a property can be preserved even by a vorticity-dissipating scheme like (4.5).

\section{Relation with "shape functions" and "nodal GLACE scheme"}

The definition (4.1) of the nodal "discrete gradient" is equivalent to the one given in $[13, \S 3.1]$, which relies on so-called "shape functions". Hereafter, we 
propose an alternative derivation of these functions, in order to show existing links with both the "symmetric potential schemes" of $[19,20]$ and the "nodal formulation" in $[5, \S 3.3]$.

7.1 A nodal definition of "curl"

The expression $\widetilde{\operatorname{curl}}_{i, j}^{(*)}$ given in [13, eqn. (3.4)] is a discrete approximation of the "curl" operator at any cell's center $x_{i}, y_{j}$; it's a linear form $\left(\mathbb{R}^{2}\right)^{9} \rightarrow \mathbb{R}(9$ corresponds to the stencil and 2 is the dimension of a velocity vector), which null-space is an hyperplane. An alternative is a "nodal curl",

$$
\begin{aligned}
\widehat{\operatorname{curl}}_{i-\frac{1}{2}, j+\frac{1}{2}}: \quad\left(\mathbb{R}^{2}\right)^{4} & \rightarrow \mathbb{R} \\
\mathbf{u}=(u, v) \mapsto \frac{1}{\Delta x}( & \left(\frac{v_{i, j+1}+v_{i, j}}{2}-\frac{v_{i-1, j+1}+v_{i-1, j}}{2}\right) \\
& \left.-\left(\frac{u_{i, j+1}+u_{i-1, j+1}}{2}-\frac{u_{i, j}+u_{i-1, j}}{2}\right)\right) .
\end{aligned}
$$

Clearly, $\widetilde{\operatorname{curl}}_{i, j}^{(*)}$ is the arithmetic average of the 4 nodal $\widehat{\operatorname{curl}}_{i \pm \frac{1}{2}, j \pm \frac{1}{2}}$, which is identical to the spherical mean as well (see $\S 4.1$ ) because the Cartesian computational grid is uniform, $\Delta x=\Delta y$, so that,

$$
\widehat{\operatorname{curl}}_{i, j}^{(*)}=\frac{1}{4}\left(\widehat{\operatorname{curl}}_{i-\frac{1}{2}, j-\frac{1}{2}}+\widehat{\operatorname{curl}}_{i-\frac{1}{2}, j+\frac{1}{2}}+\widehat{\operatorname{curl}}_{i+\frac{1}{2}, j-\frac{1}{2}}+\widehat{\operatorname{curl}}_{i+\frac{1}{2}, j+\frac{1}{2}}\right) .
$$

7.2 Derivation of these "shape functions"

The expression (4.1) of the nodal $2 \mathrm{D}$ gradient can be recast as a vector of 2 linear forms acting on the set of $2 \times 2$ matrices, $\mathcal{M}_{2,2}$, so that

$$
\frac{\partial \cdot}{\partial x} \simeq \operatorname{Tr}\left(D_{x} \cdot\right), \quad \frac{\partial \cdot}{\partial y} \simeq \operatorname{Tr}\left(D_{y} \cdot\right), \quad D_{x}, D_{y} \in \mathcal{M}_{2,2} \times \mathcal{M}_{2,2},
$$

because a standard result of linear algebra states that, for any linear form $\phi$ acting on $\mathcal{M}_{n, n}$, there exists $A_{\phi} \in \mathcal{M}_{n, n}$ such that

$$
\forall M \in \mathcal{M}_{n, n}, \quad \phi(M)=\operatorname{Tr}\left(A_{\phi} M\right) .
$$

The idea is to represent the stencil around any node as a $2 \times 2$ matrix which entries correspond to the values located at each cell's center. Accordingly, the matrices $D_{x}, D_{y}$ corresponding to the nodal gradient (4.1) read:

$$
D_{x}=\frac{1}{2 \Delta x}\left(\begin{array}{cc}
-1 & -1 \\
1 & 1
\end{array}\right), \quad D_{y}=\frac{1}{2 \Delta x}\left(\begin{array}{l}
1-1 \\
1-1
\end{array}\right) .
$$

Proposition 7.1 The "shape functions" $\hat{\Phi}$ given in [13, eqn. (3.6)] are:

$$
\mathcal{M}_{2,2} \times \mathcal{M}_{2,2} \ni \hat{\Phi}=-2 \Delta x^{2}\left(\begin{array}{c}
D_{y} \\
D_{x}
\end{array}\right) .
$$


Proof A "shape function" is indeed a collection of 4 velocity vectors in $\mathbb{R}^{2}$ which belong to the kernel of, say, $\widehat{\operatorname{curl}}_{i-\frac{1}{2}, j+\frac{1}{2}}$. Accordingly it belongs to $\mathcal{M}_{2,2} \times \mathcal{M}_{2,2}$, so let $\hat{\Phi}=(U, V)$. Moreover, from the expression (4.1),

$$
\widehat{\operatorname{curl}}_{i-\frac{1}{2}, j+\frac{1}{2}} \hat{\Phi}=\operatorname{Tr}\left(D_{x} V\right)-\operatorname{Tr}\left(D_{y} U\right),
$$

so that the "nodal curl" vanishes if $U, V$ are such that,

$$
\operatorname{Tr}\left(D_{x} V-D_{y} U\right)=0 .
$$

A sufficient condition for having a null trace is that

$$
\forall P \in \mathcal{M}_{2,2}, \quad V=D_{y} P, \quad U=D_{x} P,
$$

which is the discrete expression of "the curl of a gradient vanishes". It remains to pick $P=I d_{2}$ and to normalize with $\Delta x=\Delta y$ to reach (7.1).

If $\Delta x \neq \Delta y$, one follows Remark 4.1 in order to express the "discrete nodal gradient" by applying a rotation $R_{-\theta}$, then deduces both matrices $D_{x}, D_{y}$ and seeks $U, V \in\left(\mathcal{M}_{2,2}\right)^{2}$ which cancels the trace of each $\widehat{\operatorname{curl}}_{i \pm \frac{1}{2}, j \pm \frac{1}{2}}$.

\subsection{Resulting "curl-free" numerical scheme}

The idea proposed in [13] consists in forming a time-increment $\mathbf{u}^{n+1}-\mathbf{u}^{n} \in$ $\left[\ell^{\infty}\left(\mathbb{Z}^{2}\right)\right]^{2}$ as a linear combination of "shape functions", so as to ensure that each "nodal curl", hence the centered ones, are kept constant. Considering edge-values $p_{i, j \pm \frac{1}{2}}^{n}$ and $p_{i \pm \frac{1}{2}, j}^{n}$ given by any (approximate) 1D Riemann solver in $y$ and $x$, respectively, a standard finite-volume scheme reads,

$$
\frac{\mathbf{u}_{i, j}^{n+1}-\mathbf{u}_{i, j}^{n}}{\Delta t}=-\left(p_{i+\frac{1}{2}, j}^{n}-p_{i-\frac{1}{2}, j}^{n}\right)\left(\begin{array}{c}
\frac{1}{\Delta x} \\
0
\end{array}\right)-\left(p_{i, j+\frac{1}{2}}^{n}-p_{i, j-\frac{1}{2}}^{n}\right)\left(\begin{array}{c}
0 \\
\frac{1}{\Delta x}
\end{array}\right),
$$

which is an equality in $\mathbb{R}^{2}$ for $\Delta x=\Delta y$. By normalizing shape functions like,

$$
\hat{\Phi}=\left(\begin{array}{c}
D_{y} \\
D_{x}
\end{array}\right)=\frac{1}{2 \Delta x}\left(\begin{array}{cc}
(1,-1) & (-1,-1) \\
(1,1) & (-1,1)
\end{array}\right)
$$

one observes that, for instance,

$$
\left(\begin{array}{c}
\frac{1}{\Delta x} \\
0
\end{array}\right)=\frac{-1}{2 \Delta x}\left[\left(\begin{array}{c}
-1 \\
1
\end{array}\right)+\left(\begin{array}{c}
-1 \\
-1
\end{array}\right)\right], \quad\left(\begin{array}{c}
0 \\
\frac{1}{\Delta x}
\end{array}\right)=\frac{1}{2 \Delta x}\left[\left(\begin{array}{c}
-1 \\
1
\end{array}\right)+\left(\begin{array}{l}
1 \\
1
\end{array}\right)\right]
$$

so that each edge-value can be set as a coefficient of a linear combination of "nodal shape functions", instead of simple vectors of the canonical basis of $\mathbb{R}^{2}$. To do that, while maintaining overall consistency, let's fix one shape function 
$\hat{\Phi}$ at a particular node,say $i-\frac{1}{2}, j+\frac{1}{2}$; according to [13], its coefficient must be given by the average,

$$
\frac{1}{4}(\underbrace{p_{i-\frac{1}{2}, j}^{n}+p_{i-\frac{1}{2}, j+1}^{n}}_{\text {Riemann in } x}+\underbrace{p_{i-1, j+\frac{1}{2}}^{n}+p_{i, j+\frac{1}{2}}^{n}}_{\text {Riemann in } y}),
$$

which is exactly the "symmetric potential" denoted by $\chi_{i-\frac{1}{2}, j+\frac{1}{2}}^{n}$ in $[20$, eqn. (3.10)]. Accordingly, such a construction yields the scheme written in [20, eqn. (3.11)].

\subsection{Centered shape functions in GLACE nodal formulation}

The scheme proposed in $[5, \S 3.3]$ is originally designed on an unstructured computational grid, so the first task is to reformulate it more simply on a Cartesian grid. According to its notation, if $\Delta x=\Delta y$, the length $l_{J r}=\frac{\Delta x}{\sqrt{2}}$ and the four (nodal) normal vectors are:

$$
\forall i, j \in \mathbb{Z}^{2}, \quad \mathbf{n}_{i \pm \frac{1}{2}, j+\frac{1}{2}}=\left(\begin{array}{c} 
\pm \frac{1}{\sqrt{2}} \\
\frac{1}{\sqrt{2}}
\end{array}\right), \quad \mathbf{n}_{i \pm \frac{1}{2}, j-\frac{1}{2}}=\left(\begin{array}{c} 
\pm \frac{1}{\sqrt{2}} \\
\frac{-1}{\sqrt{2}}
\end{array}\right) .
$$

Following (7.1) and (7.2), a "shape function" is built by assembling them as an element of $\mathcal{M}_{2,2} \times \mathcal{M}_{2,2}$, which is located at the cell's center $x_{i}, y_{j}$ instead of being at each neighbor node. In any cell $J:=i, j$ of area is $\Delta x^{2}$, the semidiscrete scheme reads:

$\forall J \in \mathbb{Z}^{2}, \quad \frac{d}{d t} p_{J}+\frac{1}{\Delta x \sqrt{2}} \sum_{r}\left(\mathbf{u}_{r}, \mathbf{n}_{J r}\right)=0, \quad \frac{d}{d t} \mathbf{u}_{J}+\frac{1}{\Delta x \sqrt{2}} \sum_{r} p_{J, r} \mathbf{n}_{J r}=0$,

where each index $r$ refers to a neighbor node and fluxes are defined by solving

$$
p_{J r}=p_{J}+\left(\mathbf{u}_{J}-\mathbf{u}_{r}, \mathbf{n}_{J r}\right), \quad\left(\sum_{j} \widehat{\alpha}_{J r}\right) \mathbf{u}_{r}=\sum_{j} l_{J r} p_{j} \mathbf{n}_{J r}+\sum_{j} \widehat{\alpha}_{J r} \mathbf{u}_{J},
$$

for any fixed time $t>0$, according to the following definitions:

$$
\widehat{\alpha}_{J r}=l_{J r} \mathbf{n}_{J r} \otimes \mathbf{n}_{J r}, \quad \sum_{j} l_{J r} \mathbf{n}_{J r}=\sum_{r} l_{J r} \mathbf{n}_{J r}=0 .
$$

On a Cartesian grid, these tensorial products strongly simplify because,

$$
\begin{array}{r}
\mathbf{n}_{i-\frac{1}{2}, j-\frac{1}{2}} \otimes \mathbf{n}_{i-\frac{1}{2}, j-\frac{1}{2}}=\mathbf{n}_{i+\frac{1}{2}, j+\frac{1}{2}} \otimes \mathbf{n}_{i+\frac{1}{2}, j+\frac{1}{2}}=\frac{1}{2}\left(\begin{array}{ll}
1 & 1 \\
1 & 1
\end{array}\right), \\
\mathbf{n}_{i-\frac{1}{2}, j+\frac{1}{2}} \otimes \mathbf{n}_{i-\frac{1}{2}, j+\frac{1}{2}}=\mathbf{n}_{i+\frac{1}{2}, j-\frac{1}{2}} \otimes \mathbf{n}_{i-\frac{1}{2}, j+\frac{1}{2}}=\frac{1}{2}\left(\begin{array}{cc}
1 & -1 \\
-1 & 1
\end{array}\right),
\end{array}
$$


so that,

$$
\left(\sum_{j} \widehat{\alpha}_{J r}\right)=\sqrt{2} \Delta x\left(\begin{array}{ll}
1 & 0 \\
0 & 1
\end{array}\right):=\sqrt{2} \Delta x I d_{2} .
$$

Yet, defining a specific "nodal velocity vector", for $r=i \pm \frac{1}{2}, j \pm \frac{1}{2} \in \mathbb{Z}^{2}$,

$$
\mathbf{u}_{r}=\frac{1}{2} \sum_{J} p_{J} \mathbf{n}_{J r}+\frac{1}{2 \Delta x} \sum_{J} \widehat{\alpha}_{J r} \mathbf{u}_{J}:=\frac{\Delta x}{\sqrt{2}}(\nabla p)_{r}+\sqrt{2}<\mathbf{u}>_{r}
$$

and rewriting (7.3) as a linear combination of neighboring nodal values,

$\frac{d}{d t} p_{J}(t)+\frac{1}{\Delta x \sqrt{2}} \sum_{r}\left(\mathbf{u}_{r}, \mathbf{n}_{J r}\right)=0, \quad \frac{d}{d t} \mathbf{u}_{J}(t)+\frac{1}{\Delta x \sqrt{2}} \sum_{r} \widehat{\alpha}_{J r}\left(\mathbf{u}_{J}-\mathbf{u}_{r}\right)=0$,

the resulting value of any "nodal pressure gradient" matches again (4.1),

$$
\text { for } r=i-\frac{1}{2}, j+\frac{1}{2}, \quad(\nabla p)_{r}=\frac{1}{2 \Delta x}\left(\begin{array}{c}
p_{i, j+1}+p_{i, j}-p_{i-1, j+1}-p_{i-1, j} \\
p_{i, j+1}+p_{i-1, j+1}-p_{i-1, j}-p_{i, j}
\end{array}\right) \text {. }
$$

\section{Conclusion}

The design of accurate multi-dimensional numerical schemes for evolution equations is often difficult as simple dimensional-splitting may reveal itself insufficient. Two roadmaps co-exist to improve the existing situation:

- one consists in proceeding through "proxies", for instance the preservation of auxiliary quantities (systems with "involutions" in Dafermos' terminology) like vorticity or divergence constraints, see [13,18-20];

- another, in starting from the expression of a multi-D (possibly local) solution, then deducing a numerical algorithm, in the spirit as e.g. $[11,12]$ for parabolic equations, or [4]. This is what is done here, when Kirchhoff's exact solution yields a time-marching scheme for (1.1), in the form (1.2), see [27].

Acknowlegments We thank Prof. Philip L. Roe for stimulating discussions.

\section{References}

1. D. Amadori, L. Gosse, Error Estimates for Well-Balanced Schemes on Simple Balance Laws, BCAM SpringerBriefs in Mathematics, ISBN: 978-3-319-24784-7 (2015)

2. B. Bidégaray, J.M. Ghidaglia, Multidimensional corrections to cell-centered finite volume methods for Maxwell equations, Appl. Numer. Math., 44 (3), 281-298, 200

3. W. Barsukow, Ch. Klingenberg, Exact solution and a truly multi-dimensional Godunov scheme for the acoustic equations, preprint (2017)

4. M. Brio, A.R. Zakharian, G.M. Webb, Two-dimensional Riemann solver for Euler equations of gas dynamics, J. Comput. Phys. 167 (2001) 177-195

5. C. Buet, B. Després, E. Franck, Design of asymptotic preserving finite volume schemes for the hyperbolic heat equation on unstructured meshes, Numer. Math. (2012) 122: 227278 . 
6. Davis, S: A rotationally biased upwind difference scheme for the Euler equations, J. Comput. Phys. 56, (1984), 65-92.

7. L.C. Evans, Partial Differential Equations, 2nd Edition, Grad. Texts in Math. 19-R (2010) Amer. Math. Soc., ISBN: 978-0-8218-4974-3.

8. Doreen Fan, P.L. Roe, Investigations of a New Scheme for Wave Propagation, Proceedings of 22nd AIAA Computational Fluid Dynamics Conference (22-26 June 2015, Dallas, TX), DOI: $10.2514 / 6.2015-2449$.

9. E.C. Gartland, Jr., Discrete weighted-mean approximation of model convection-diffusion equation, SIAM J. Sci. Stat. Comp. 3 (1982) 460-472.

10. L. Gosse, A two-dimensional version of the Godunov scheme for scalar balance laws, SIAM J. Numer. Anal. 52 (2014), no. 2, 626-652

11. L. Gosse, Viscous Equations Treated with $\mathcal{L}$-Splines and Steklov-Poincaré Operator in Two Dimensions, to appear (2017) DOI: 10.1007/978-3-319-49262-9_6.

12. L. Gosse, Dirichlet-to-Neumann mappings and finite-differences for anisotropic diffusion, Comput. \& Fluids 156 (2017) 58-65.

13. R. Jeltsch, M. Torrilhon, On curl-preserving finite volume discretizations for shallow water equations, BIT 46 (2006) 35-53.

14. P.D. Lax, B. Wendroff, Difference schemes for hyperbolic equations with high order of accuracy, Comm. Pure Appl. Math. 17 (1964), 381-398.

15. A. Lerat, F. Falissard, J. Sidés, Vorticity-preserving schemes for the compressible Euler equations, J. Comput. Phys. 225 (2007) 635-651.

16. J. Li, M. Lukacova, G. Warnecke, Evolution Galerkin schemes applied to twodimensional Riemann problems for the wave equation system, DCDS-B 9 (2003) 559-576

17. Jiequan Li, Tong Zhang, Shuli Yang, The Two-Dimensional Riemann Problem in Gas Dynamics, Pitman Monographs and Surveys in Pure and Applied Mathematics (1998) ISBN: 0582244080

18. Tyler Lung, P.L. Roe, Toward a reduction of mesh imprinting, Intern. J. Numer. Meth. Fluids 76 (2014) 450-470.

19. S. Mishra, E. Tadmor, Vorticity Preserving Schemes Using Potential-Based Fluxes for the System Wave Equation, in Hyperbolic problems: theory, numerics and applications, Proc. Sympos. Appl. Math. 67 (2009) 795-804.

20. S. Mishra, E. Tadmor, Constraint-preserving schemes using potential-based fluxes. II. Genuinely multi-dimensional systems of conservation laws, SIAM J. Numer. Anal. 49 (2011) 1023-1045.

21. K.W. Morton, P.L. Roe, Vorticity preserving Lax-Wendroff type schemes for the system wave equation, SIAM. J. Sci. Comput. 23 (2001) 170-192.

22. B. Parlett, Accuracy and dissipation in difference schemes, Comm. Pure Appl. Math. 19 (1966) 111-123.

23. M.H. Pham, M. Rudgyard, E. Süli, Bicharacteristic methods for multi-dimensional hyperbolic systems, in Godunov methods: theory and applications, E.F. Toro Editor (Kluwer Academic/Plenum, New York) 2001.

24. J. Rauch, BV estimates fail most quasilinear hyperbolic systems in dimensions greater than one, Commun. Math. Phys. 106 (1986) 481-484.

25. Roe, P. L.: Discrete Models for the Numerical Analysis of Time- Dependent Multidimensional Gas Dynamics, J. Comput. Phys. 63, (1986), 458-476.

26. Roe, P. L.: Discontinuous Solutions to Hyperbolic Systems Under Operator Splitting, Numer. Meth. in P.D.E. 7, (1991), 277-297.

27. Roe, P. L.: Is Discontinuous Reconstruction Really a Good Idea?, J. Scient. Comput. 73, (2017), 1094-1114.

28. B. Wendroff, A Two-Dimensional HLLE Riemann Solver and Associated Godunov-Type Difference Scheme for Gas Dynamics, Comput. \& Math. Applic. 38 (1999) 175-185

29. Yuxi Zheng, Systems of Conservation Laws: Two-Dimensional Riemann Problems, Progress in Nonlinear Differential Equations and Their Applications 38 (Birkhauser Verlag)

30. Y. Zheng, Z. Robinson, The pressure-gradient system, Meth. Applic. Anal. 17 (2010) $263-278$. 